\title{
O APRENDIZADO DE ALGORITMOS COMO FERRAMENTA DO ENGENHEIRO DO AMANHÃ: DESENVOLVIMENTO DE APLICATIVOS MÓVEIS PARA INSTIGAR O USO DA COMPUTAÇÃO
}

Cádmo A. R. Dias-cadmordias@gmail.com

Lidyane F.da Silva - lidyane.control.aut@gmail.br

Caio I. V. Nazareth - caioigvasconcelos@gmail.com

Renata U. Rêgo - renata.umbelino@pucminas.br

Pontifícia Universidade Católica de Minas Gerais (PUC Minas)

Rua Dom José Gaspar, 500, Coração Eucarístico

30535-901- Belo Horizonte - Minas Gerais

Resumo: Na área da Engenharia, a constante utilização dos métodos computacionais já se torna necessária e faz parte das tecnologias de informação presentes cada dia mais da Indústria 4.0, cenário vivido pelos recém e futuramente profissionais formados na área. Participante da ementa da maioria dos cursos de Engenharia do país, disciplinas de programação de computadores que trabalham raciocínio lógico, algoritmos e linguagens de programação não são de domínio de todos os alunos e, diversas vezes, estes não se interessam pelo conteúdo. Entretanto, a utilização de softwares se torna cada vez mais frequente na área, uma vez que estes proporcionam resultados mais precisos, confiáveis e rápidos, reduzindo drasticamente o tempo de projeto. Portanto, o futuro engenheiro que pratica a interdisciplinaridade com conceitos de Computação e Cálculo, se forma com maiores capacidades para o desenvolvimento de raciocínio lógico e para a solução de problemas. Nesse contexto, o artigo em questão busca apresentar os resultados de uma pesquisa feita com estudantes de Engenharia de áreas, faixas etárias e experiências profissionais distintas, mostrando as dificuldades enfrentadas pelos mesmos e o que estes pensam sobre o conteúdo, sendo uma barreira na formação do engenheiro do amanhã. Além de tal, o presente artigo busca apresentar diversos trabalhos que vêm sendo realizados integrando o desenvolvimento de aplicativos móveis e softwares para aplicações em Engenharia.

Palavras-chave: Programação na Engenharia. Algoritmos. Rotinas de programação. Softwares. Aplicativos Android.

\section{INTRODUÇÃO}

Desde o surgimento dos primeiros dispositivos computacionais, datados por volta da década de 1940, a Computação vem se desenvolvendo em nível exponencial e auxiliando na atuação das mais diversas áreas profissionais. De acordo com Filho (2007), foi a partir de 1950 que se observou o avanço da Ciência da Computação em extensão e profundidade. Ainda de acordo com o autor em questão, essa foi a época em que se iniciaram as diversas transformações não apenas nas áreas de desenvolvimento de hardwares e softwares, mas também na criação de algoritmos por meio das linguagens de programação. 
Nos estudos mais recentes que dizem respeito ao caminho que a Engenharia deve tomar nos próximos anos e por consequência os futuros engenheiros, muito se fala de termos como Internet das Coisas, Indústria 4.0 e Aprendizagem de Máquina, por exemplo. Em meio a todo o avanço tecnológico que os termos apresentados trazem, os atuais currículos de Engenharia do país contam com disciplinas que trabalham o desenvolvimento de algoritmos e que são base para o enveredamento nas áreas supracitadas. Entretanto, observa-se que frequentemente o ensino dessas disciplinas não são bem aproveitadas pelos alunos, de maneira que estes não buscam o profundo aprendizado e a aplicação dos conceitos em outras áreas e disciplinas, tornando-se uma barreira de hoje na formação do engenheiro do amanhã (MAGRANI, 2018; MATULOVIC et al., 2020; OLIVEIRA E SIMÕES, 2017).

Como apresentado por Araújo et al. (2017), grande parte dos atuais discentes de Engenharia são nascidos e formados na era digital. Tal apontamento significa dizer que os dispositivos móveis têm grande audiência e são objetos indispensáveis tanto no cotidiano pessoal quanto no cotidiano profissional. Com isso, a utilização desses recursos em sala de aula pode não apenas ser uma ferramenta para inovar na relação ensino-aprendizado, mas também uma solução para já formar e inserir o estudante de Engenharia nas habilidades que serão extremamente necessárias para o futuro profissional.

Portanto, no contexto exposto, o artigo em questão tem como objetivo principal investigar as questões apresentadas sob o ponto de vista do pensamento de alunos de graduação em cursos de Engenharia, além de apresentar trabalhos atuais que utilizam a programação de computadores de maneira prática e que atraiam a atenção dos alunos de Engenharia. Com o intuito de obter resultados que tivessem maior representatividade, uma pesquisa foi realizada com cerca de 50 alunos de aproximadamente 10 áreas distintas da Engenharia, além de faixas etárias e experiências profissionais diversificadas. Uma aplicação para dispositivos Android que auxilia no ensino e desenvolvimento de projetos também é apresentada.

\section{O ENSINO DE PROGRAMAÇÃO DE COMPUTADORES NA ENGENHARIA}

Seja o termo algoritmo definido como um conjunto de instruções que serão realizadas, em etapas, sequenciadas, o qual possui uma lógica a ser seguida. Esta lógica nada mais é do que o método que será utilizado e implementado buscando solucionar o problema com um melhor desempenho. Muitas vezes comparado a uma receita de bolo, Lung (2007) diz que existem diversos tipos de algoritmos, sendo eles o Pseudocódigo, parecido ao português estruturado; a Descrição Narrativa, que faz uso de uma linguagem natural; o Fluxograma, frequentemente utilizado nos diversos setores e também conhecido como diagrama de blocos; e o Diagrama de Chapin, que organiza o problema através de um diagrama de quadros e que apresenta uma visão hierárquica e estruturada. Para Santos (2020), no entanto, o exercício de escrever um algoritmo envolve muito mais do que apenas escrever uma receita de bolo a ser seguida passo a passo, se tornando uma tarefa um tanto quanto árdua em alguns casos.

O aprendizado de algoritmos e da programação de computadores é previsto na ementa curricular dos cursos de Engenharia do país, comumente passando por linguagens como Portugol, Linguagem $\mathrm{C}$ e $\mathrm{C}++$, Fortran e linguagens de domínio específico próprio (DSL) de outros programas, como o comumente utilizado MATLAB. Entretanto, devido à problemática apresentada acima pela observação de Santos (2020), o aprendizado das disciplinas em questão pode ser deixado de ser aproveitado por muitos alunos da graduação quando estes não se interessam pela programação de computadores. Contudo, esse pode ser um problema maior quando, analisando os rumos que a Engenharia vem tomando, observa-se que esta está cada 
vez mais ligada à Computação. Corroborando com tal, Monteiro (2009) diz que as calculadoras substituíram a régua de cálculo, além de que os softwares conduzem a Engenharia. Seguindo essa mesma linha de raciocínio e analisando a importância da programação atualmente, para a Sociedade Brasileira de Computação (SBC) o pensamento computacional é o processo cognitivo usado pelos seres humanos para resolver problemas, habilitando aos estudantes para a organização de soluções de problemas por meio de recursos como abstração, refinamento, modularização dentre outros (SBC, 2016).

O curso de programação de computadores possui um papel importante na formação dos alunos. De acordo com Ozyurt e Ozyurt (2018), este curso tem como objetivo ensinar alguns conhecimentos básicos sobre algoritmos, apresentar uma linguagem de programação e de auxiliar os alunos a desenvolverem habilidades de lógica de programação. Desta forma, o conteúdo abordado durante o curso abrange temas introdutórios, os quais apresentam operações de entrada e saída, estruturas condicionais, estruturas de repetição, matrizes unidimensionais e bidimensionais, apresentação de algoritmos de ordenação e algoritmos de busca, além de apresentar e fazer uso de funções iterativas e recursivas. Os autores comentam, ainda, que a disciplina de programação é considerada difícil pelos alunos nos primeiros anos de formação universitária, onde os alunos apresentam uma atitude/percepção negativa e baixa motivação, sendo estes os problemas mais comuns entre os estudantes.

No cenário apresentado, pode-se concluir que estes problemas influenciam fortemente no desempenho dos alunos, pois resultam em um baixo aproveitamento dos mesmos, fazendo com que desenvolvam poucas habilidades lógicas por meio desta disciplina. A dificuldade na compreensão da programação de computadores vem sendo foco de estudos em todo mundo a alguns anos, e no caso do Brasil, dados mostram que durante algum tempo o foco dos estudos esteve na dificuldade de assimilação das abstrações presentes no processo de aprendizagem (PEREIRA JÚNIOR E RAPKJEWICZ, 2004).

No entanto, a programação pode ser aprendida mais facilmente graças às modernas abordagens educacionais, como os ambientes de aprendizagem centrados no aluno e a aprendizagem ativa. A partir deste princípio, Ozyurt e Ozyurt (2018) utilizam a abordagem Flipped Classroom (FCA) como solução para os problemas apresentados. Esta abordagem é baseada na inversão dos papéis das salas de aula e das casas dos estudantes. Neste contexto, o aluno inicialmente estuda em casa com o intuito de preparar-se para participar das atividades em sala e, em sala de aula, o aluno pratica os conceitos vistos anteriormente em atividades práticas e esclarece suas dúvidas com seu professor. Após a aula, o aluno consulta seu desempenho exercido em sala. A partir do feedback apresentado pelo professor, o aluno revisa o conteúdo buscando aprimorar os novos conhecimentos adquiridos. Os autores em questão apresentam, ainda, que apesar dos resultados efetivos e produtivos apresentados em vários aspectos, a FCA também apresenta aspectos negativos. Porém, apesar dos aspectos negativos, os autores concluem que este modelo tem apresentado uma melhora significativa na aprendizagem de programação dos alunos de Engenharia.

\section{A VISÃO DOS ESTUDANTES DE ENGENHARIA SOBRE ALGORITMOS}

Por meio de um serviço on-line de criação de questionários, algumas questões foram propostas. Dentre elas, algumas do tipo múltipla escolha continham possibilidades de resposta que variavam entre as opções "muito", "mediano" e "pouco". Outras questões como faixa etária e curso de graduação em que o aluno se encontra matriculado foram realizadas, além de uma questão aberta para que o participante sugerisse o que poderia ser melhor no processo de ensino-aprendizado das respectivas disciplinas. Estando o questionário disponível para 
Dos alunos entrevistados, o aprendizado de algoritmos é visto positivamente em outros campos. Sendo unanimidade o fato de que aprender programação ajudou no desenvolvimento do raciocínio lógico para as diversas questões internas e externas à academia, apenas 9,6\% dos alunos disseram que não foi possível realizar a interdisciplinaridade com esse conteúdo.

Buscando trabalhar com dados mais amplos, questões como contato com práticas de programação, idade, universidade em que realiza o curso de Engenharia e questões afins também foram realizadas. Com isso, uma importante condição de contorno a ser ressaltada é de que $69,2 \%$ dos estudantes nunca tiveram contato com desenvolvimento de rotinas e programação antes do curso de graduação, o que poderia dificultar o desenvolvimento das habilidades. Entretanto, um fator que poderia facilitar tal questão é que $88,5 \%$ dos entrevistados têm idade entre 20 e 30 anos. Apesar da maioria dos estudantes que responderam o questionário serem dos cursos de Engenharia Mecatrônica e Engenharia Mecânica, também participaram do formulário estudantes de Engenharia Civil, Química, de Controle e Automação, Metalúrgica e de Produção. O Quadro 1 apresenta todas as questões presentes no formulário.

Quadro 1-Questões realizadas aos estudantes de engenharia

\begin{tabular}{|c|c|}
\hline Questão & Opções de resposta \\
\hline Curso de engenharia sendo realizado & $\begin{array}{l}\text { Alimentos, Ambiental, Civil, Controle e } \\
\text { Automação, Mecânica, Mecatrônica, } \\
\text { Metalúrgica, Produção, Química, outras }\end{array}$ \\
\hline Faixa etária (em anos) & $<20,20-30,31-40,41-50,>50$ \\
\hline Endereço eletrônico (e-mail) & Questão aberta \\
\hline Em qual universidade você estuda? & Questão aberta \\
\hline $\begin{array}{l}\text { Previamente à disciplina, você teve contato } \\
\text { com programação de computadores? }\end{array}$ & Sim, não \\
\hline $\begin{array}{l}\text { Em sua opinião, engenharia e computação } \\
\text { tendem a um caminho indissociável? }\end{array}$ & Sim, não, em partes \\
\hline $\begin{array}{c}\text { Em sua opinião, o aprendizado de técnicas } \\
\text { de computação é fundamental para a } \\
\text { formação do engenheiro do amanhã? }\end{array}$ & Sim, não, em partes \\
\hline $\begin{array}{l}\text { As disciplinas de algoritmos te ajudaram a } \\
\text { desenvolver o raciocínio lógico? }\end{array}$ & $\begin{array}{l}\text { Sim, um pouco, poderia ser melhor } \\
\text { aproveitado, não }\end{array}$ \\
\hline $\begin{array}{l}\text { O conteúdo aprendido nas disciplinas de } \\
\text { programaçãa ajudou em outras disciplinas } \\
\text { da universidade e em tarefas diárias? }\end{array}$ & $\begin{array}{l}\text { Sim, um pouco, poderia ser melhor } \\
\text { aproveitado, não }\end{array}$ \\
\hline $\begin{array}{l}\text { Sugestões para a melhoria do aprendizado } \\
\text { nas disciplinas de programação (opcional) }\end{array}$ & Questão aberta \\
\hline
\end{tabular}

Fonte: Elaborado pelos autores.

\section{O DESENVOLVIMENTO DE APLICATIVOS MÓVEIS E SOFTWARES PARA APLICAÇÕES DE ENGENHARIA}

No cenário até então apresentado e com a redução da dificuldade de acesso aos recursos para o desenvolvimento de softwares e aplicativos móveis, a criação por meio de recursos solicitados nos dias de hoje e a utilização destes nas mais diversas áreas e tarefas da Engenharia tem se difundido e se tornado primordial ferramenta (ROCHA, 2019). O interessante de observar é que, anteriormente desenvolvido em grande maioria por estudantes 
e profissionais da Computação, hoje em dia muitos trabalhos resultantes em softwares e aplicativos móveis estão sendo desenvolvidos por alunos de Engenharia. Com isso, há uma categoria própria de softwares para aplicações em Engenharia. (PRESSMAN E MAXIM, 2016).

No contexto apresentado que Lima e Violin (2019) desenvolveram um aplicativo para que estudantes de Engenharia Civil possam ampliar seus conhecimentos sobre as situações e conceitos que envolvem cada etapa de uma obra, além de incentivá-los a se tornarem alunos ativos no próprio processo de aprendizagem. Na mesma área, Dias et al. (2018) desenvolveram um outro aplicativo para dispositivos Android responsável pela classificação de solos. Com parâmetros inicialmente obtidos de experimentos em campo, o usuário do aplicativo consegue obter o tipo de solo de acordo com os métodos Transportation Research Board (TRB) e Unified Soil Classification System (USC). Vaz (2018) apresenta os aplicativos de uma maneira mais lúdica ao desenvolver um aplicativo para o ensino de Engenharia de Produção por meio de um jogo. Enquanto Lamy (2018) produz em um aplicativo computacional para a função perda de qualidade, Silva (2019) trabalha no aperfeiçoamento de um aplicativo para dispositivo móvel orientado a gestão de projetos de recuperação de áreas degradadas. Por outro lado, Lima e Santos (2019) buscam utilizar um aplicativo móvel para celular no ensino do projeto de câmaras de separação gravitacional, onde como um dos resultados o usuário consegue um gráfico de eficiência de coleta de acordo com o diâmetro da amostra. Na área de fluidos, Braga et al. (2017) abordam o desenvolvimento de uma aplicação Android para solução de questões relativas à hidráulica de canais ou condutos livres. O interessante do presente caso é que o mesmo se apresenta como uma ferramenta didática para o ensino das disciplinas dos cursos de Engenharia que abordam os relativos conceitos de hidráulica. Da mesma forma, apresentando um lado didático com conceitos teóricos inclusos para o usuário, outros programas com auxílio do software MATLAB foram desenvolvidos, como por exemplo um para projetos do mecanismo came-seguidor, um dos clássicos dispositivos de aplicações mecânicas como motores (DIAS, VIMIEIRO e MAIA, 2018).

Como relatado pelos trabalhos supracitados, o desenvolvimento de aplicativos que abordam as teorias de Engenharia ajuda não apenas no aprendizado dos conhecimentos técnicos das respectivas áreas, mas também na utilização dos conceitos computacionais em outras tarefas. No momento em que o aluno aplica conceitos algorítmicos como estruturas de repetição e condicionamento, por exemplo, na solução de um problema, o mesmo começa a observar a aplicação desses conceitos na solução de outras questões e por consequência torna a programação de computadores uma ferramenta para seu dia-a-dia.

De maneira a corroborar com o exposto no presente capítulo, este trabalho apresenta, também, um outro aplicativo para dispositivos Android que está sendo desenvolvido por alunos de Engenharia e Computação da Pontifícia Universidade Católica de Minas Gerais (PUC Minas). Novamente, a motivação da elaboração desse aplicativo é o exercício da utilização de algoritmos para soluções de Engenharia. O aplicativo busca auxiliar no cálculo de projetos e no aprendizado dos principais parâmetros de três dos principais tipos de engrenagens: Engrenagem de dentes retos, helicoidais e cônicos. A Figura 3 apresenta a tela inicial do programa e entrada de parâmetros para o projeto de uma engrenagem. 
Figura 3 - Telas principais do aplicativo para cálculo de engrenagens.

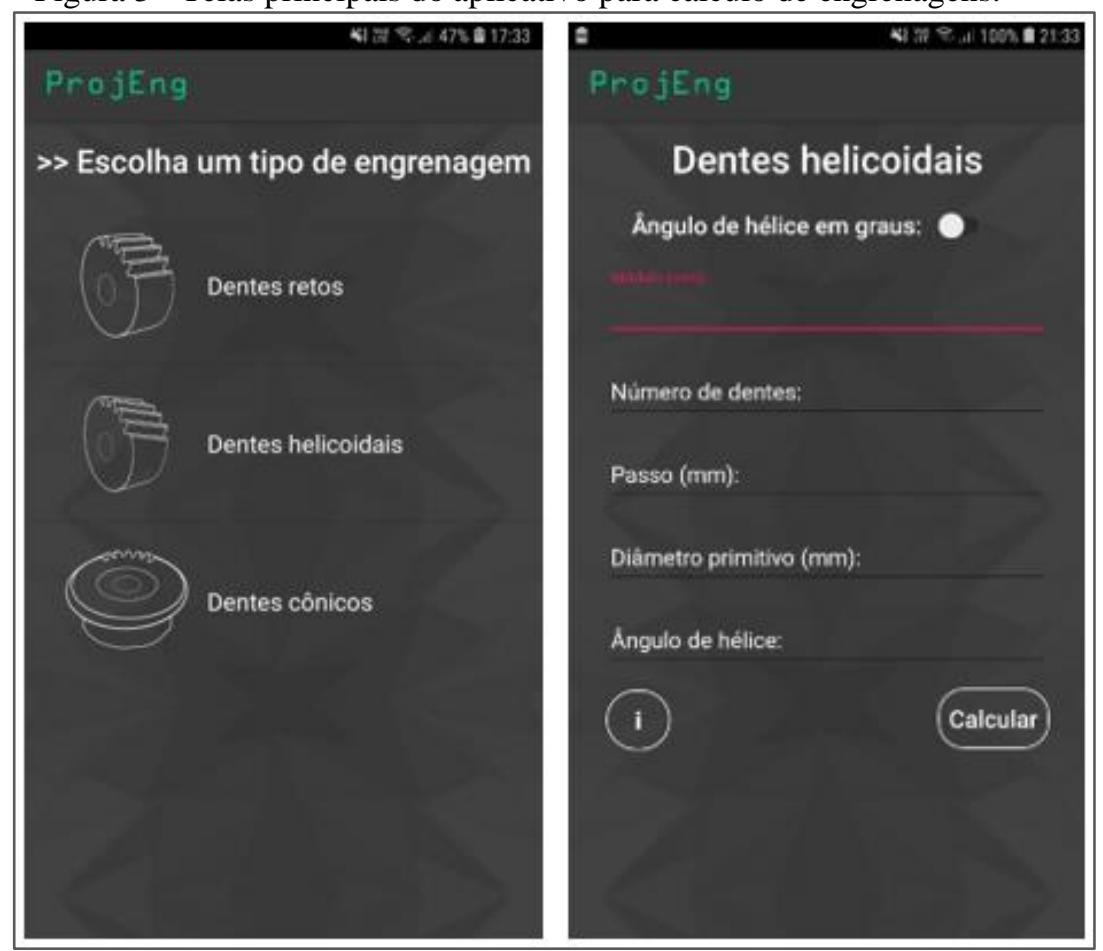

Fonte: Elaborado pelos autores

No menu principal acima apresentado à esquerda, é possível escolher qual dos três tipos de engrenagem é desejado realizar o cálculo. Uma vez selecionado, outro menu será apresentado como disponível à direita da Figura 3, permitindo ao usuário a entrada de dados.

O interessante de ser observado na última figura apresentada é que há, ainda, um botão de informação para cada tipo de engrenagem. Quando o usuário seleciona essa opção, ele poderá ver informações relativas ao tipo de engrenagem, ou seja, onde e o porquê da aplicação de cada engrenagem, suas vantagens e desvantagens, detalhes acerca dos principais parâmetros e etc., servindo como uma boa ferramenta para o aprendizado de disciplinas relacionadas nos cursos de Engenharia. Ressaltando que engrenagens são componentes extremamente utilizados em diversas aplicações da mecânica, o aplicativo apresentado não será profundamente abordado por ele não ser foco do presente trabalho, mas sim mais um caso de exemplo para corroborar com a importância da utilização dos recursos computacionais e conceitos algorítmicos como ferramentas do engenheiro do amanhã.

\section{CONSIDERAÇÕES FINAIS}

Não é novidade que, com o passar do tempo, cada vez mais a Computação tem se tornado uma ferramenta auxiliadora para a Engenharia. Reduzindo o tempo de projeto, as diversas funcionalidades trazidas por softwares têm proporcionado resultados mais precisos e confiáveis. Entretanto, para a utilização de tal, além da importância de um conhecimento bem fundamentado em teorias, o aluno que está se graduando em Engenharia, seja qual for ela, necessita de uma formação concisa em que a utilização de softwares não seja algo de desconhecimento, mas sim uma ferramenta a ser frequentemente utilizada.

Entretanto, como foi possível analisar pelas discussões das entrevistas com cerca de 50 graduandos de Engenharia, grande parte dos alunos, quando questionada do que poderia ter 
sido melhor durante o ensino das disciplinas respectivas, apresentaram questões relativas à necessidade de exemplos mais diretos e métodos mais dinâmicos, de maneira a aplicar o desenvolvimento de rotinas na prática da Engenharia.

Quanto ao que vem sendo realizado atualmente, diversos trabalhos mostraram a utilização de algoritmos para o desenvolvimento de aplicativos Android na área da Engenharia. Enquanto alguns têm foco em aplicações diretas e práticas para soluções de problemas em campo, outras buscam focar na relação ensino-aprendizagem e até mesmo propõe jogos para tal. Observou-se, ainda, que em especial no caso destes últimos, o grande foco é tornar mais interessante e por consequência atrair a atenção de alunos de Engenharia para o conhecimento dos recursos de computação, uma ferramenta fundamental para o engenheiro do amanhã.

O trabalho em questão apresentou, também, outro aplicativo desenvolvido em sistema Android para o aprendizado de projetos de engrenagens. Com isso, foi possível concluir, portanto, que em um mundo onde cada vez mais a Engenharia utiliza os artifícios da Computação é de fundamental importância que os conceitos e incentivo ao desenvolvimento de algoritmos, rotinas de programação e até mesmo softwares e aplicativos sejam realizados no meio acadêmico. Corroborando com as ideias apresentadas, Roque et al. (2017) comenta que a formação do perfil do engenheiro deve ser tal que capacite o futuro profissional a desenvolver o raciocínio lógico para a resolução de problemas, sendo o conhecimento em programação de computadores um meio facilitador para que o objetivo em questão seja alcançado.

\section{Agradecimentos}

Os autores agradecem à Pontifícia Universidade Católica de Minas Gerais (PUC Minas) pelo suporte. Este estudo foi financiado em parte pela Coordenação de Aperfeiçoamento de Pessoal de Nível Superior - Brasil (CAPES) - Código Financeiro 001.

\section{REFERÊNCIAS}

ARAÚJO, Gracielle A.; PINHEIRO, Váleria R.; SANTOS, Adelino J. Análise da percepção dos aplicativos móveis no processo de ensino aprendizagem da matemática no ensino superior. In: VII Congresso Internacional de Ensino de Matemática, 2017, Canoas (RS). Anais.

BRAGA, P. H. A.; MENEZES FILHO, F. C. M.; FREIRE, G. M. Desenvolvimento de um aplicativo Android para a hidráulica de canais. Revista Eletrônica de Engenharia Civil, v. 13, n.1, p. 297-303, 2017.

DIAS, Cádmo A. R. et al. Promovendo o ensino em engenharia por meio da interdisciplinaridade: desenvolvimento de um software para o ensino de mecânica dos solos. In: XLVI Congresso Brasileiro de Educação em Engenharia e $1^{\circ}$ Simpósio Internacional de Educação em Engenharia, 2018, Salvador (BA). Anais.

DIAS, Cádmo A. R.; VIMIEIRO, Claysson B. S.; MAIA, Luis H. A. Inovando o ensino por meio da interdisciplinaridade: desenvolvimento de uma rotina de programação, em MATLAB, para o estudo do mecanismo came seguidor. In: XLVI Congresso Brasileiro de Educação em Engenharia e $1^{\circ}$ Simpósio Internacional de Educação em Engenharia, 2018, Salvador-BA. Anais. 
FILHO, Cléuzio Fonseca. História da Computação: O caminho do pensamento e da tecnologia. 1. ed. Porto Alegre: EDIPUCRS, 2007.

LAMY, Lucas. Aplicativo computacional para a função perda de qualidade, razão sinal-ruído e análise experimental de Taguchi. 2018. 50 f. Dissertação (Mestrado) - Universidade Federal do Paraná, Curitiba, 2018.

LIMA, G. M.; SANTOS, K. D. O uso de um aplicativo móvel para celular no ensino do projeto de câmaras de separação gravitacional. In: XXXIX Congresso Brasileiro de Sistemas Particulados, 2019, Belém-PA. Anais.

LIMA, K. M.; VIOLIN, R. Y. T. Gamificação aplicada ao processo de aprendizagem no curso de Engenharia Civil. In: XI Encontro Internacional de Produção Científica, 2019, MaringáPR. Anais.

LUNG, Lau Cheuk. Introdução a Algoritmos. Disponível em: http://www.inf.ufsc.br/ lau.lung/INE5201/Aula\%204\%20-\%20Introducao_Algoritmo.pdf. Acesso em: 30 jul. 2020.

MAGRANI, Eduardo. A Internet das Coisas. Rio de Janeiro: FGV Editora. 2018.

MATULOVIC, M. et al. Desenvolvimento de uma smart house no curso de Engenharia de biossistemas. Brazilian Journal of Biosystems Engineering, v. 14, n.1, p. 48-61, 2020.

SANTOS, Luis Carlos de Castro. Introdução à Lógica de Programação e Modelagem Computacional. Disponível em: https://edisciplinas.usp.br/pluginfile.php/5147516. Acesso em: 17 set. 2020.

MONTEIRO, Marcus. Softwares transformam a Engenharia estrutural. Disponível em: http://www.cimentoitambe.com.br/softwares-transformam-a-engenharia-estrutural/. Acesso em: 30 jul. 2020.

OLIVEIRA, F. T.; SIMÕES, W. L. A indústria 4.0 e a produção no contexto dos estudantes da Engenharia. In: Simpósio de Engenharia de Produção, 2018, Catalão-GO. Anais.

OZYURT, Hacer and OZYURT, Ozcan. Analyzing the effects of adapted flipped classroom approach on computer programming success, attitude toward programming, and programming self-efficacy. Computer Applications in Engineering Education, v. 26, n.6, p. 2036-2046, 2018.

PEREIRA JÚNIOR, José C. R.; RAPKIEWICZ, C. E. O Processo de Ensino e Aprendizagem de Algoritmos e Programação: Uma Visão Crítica da Literatura. In: III Workshop de Educação em Computação e Informática do Estado de Minas Gerais, 2004, Belo HorizonteMG. Anais.

PRESSMAN, R.; MAXIM, B. Engenharia de Software: Uma abordagem profissional. Nova Iorque: McGraw Hill. 2016. 
ROCHA, Gabriel da S. C. Engenharia de Software: aplicativos web progressivos nativos para o comércio utilizando inteligência artificial para tomada de decisões. 2019. Tese (Graduação) - Faculdade Anhanguera, São Paulo, 2019.

ROQUE, Gabriel Rocha et al. Experimentação remota no ensino de superior: Linguagens de programação nas Engenharias Mecatrônica e Automação Industrial. Revista de Ensino de Engenharia, Brasília, v.36, n.2, p. 789-799, 2017.

SBC, Sociedade Brasileira de Computação (2016). Computação na Base Nacional Comum Curricular. Elaborada pela comissão de Educação da SBC em colaboração com a Comissão Especial de Informática na Educação e membros da SBC.

SILVA, Roney D. C. Aperfeiçoamento de aplicativo para dispositivo móvel orientado a gestão de projetos de recuperação de áreas degradadas. 2019. Tese (Graduação) Universidade Federal de Mato Grosso, Mato Grosso, 2019.

VAZ, Antônio C. da S. B. Desenvolvimento de um jogo digital para ensino de engenharia de produção. 2018. 50 f. Tese (Graduação) - Universidade Federal de Campina Grande, Paraíba, 2019.

\title{
LEARNING FROM ALGORITHMS AS A TOOL FOR TOMORROW ENGINEER: DEVELOPING MOBILE APPLICATIONS TO INSTIGATE COMPUTER SCIENCE USE
}

\begin{abstract}
In Engineering, the constant use of computational methods is already essential and is part of the composition of Industry 4.0, a scenario experienced by recently and future professionals trained in the area. Participant in most of engineering courses in the country, computer programming disciplines that work with logical reasoning, algorithms and programming languages are not the domain of all students and, many times, they are not interested in the content. However, the use of software is becoming more and more frequent in the area, since they provide more accurate, reliable and fast results, drastically reducing the design time. Therefore, the future engineer who practices interdisciplinarity with concepts of Computing is trained with greater capacities for the development of logical reasoning and for the solution of problems. In this context, the article in question aims to present the results of a research carried out with engineering students from different areas, age groups and professional experiences, showing the difficulties faced by them and what they think about the content, being a barrier in the formation of the engineer of tomorrow. In addition, this article seeks to present several works that have been carried out integrating the development of mobile applications and software for applications in Engineering.
\end{abstract}

Keywords: Engineering and Programming. Algorithms. Programming routines. Software. Android Applications. 